\title{
AVANĢOS E DESAFIOS PARA A IMPLEMENTAÇÃO DAS PRÁTICAS INTEGRATIVAS E COMPLEMENTARES NO BRASIL
}

Advances and challenges for the implementation of integrative and complementary praetices in Brazil

\author{
Bárbara Oliveira Reis ${ }^{1}$, \\ Larissa Rodrigues Esteves ${ }^{2}$, Rosangela Maria $\mathrm{Greco}^{3}$
}

\begin{abstract}
RESUMO
Analisou-se a conjuntura da produção científica, os avanços e os desafios para a implementação das Práticas Integrativas e Complementares (PIC) no Brasil. O estudo se deu por meio de Revisão Integrativa dos artigos indexados nas bases de dados bibliográficas da Rede Biblioteca Virtual de Saúde (BVS), selecionando-se 68 artigos. Notou-se que a região Sudeste permanece como polo científico e a maior parte dos estudos não possui nível de evidência significativo. No decorrer dos anos, houve pequeno aumento no número de estudos e maior comprovação da eficácia e eficiência das PIC em diversas áreas, porém entraves relacionados a pouca formação profissional, má gestão do Sistema e concepções enraizadas na medicina alopática permanecem e dificultam a ampliação das PIC no país.
\end{abstract}

PALAVRAS-CHAVE: Antroposofia; Fitoterapia; Homeopatia; Medicina Tradicional Chinesa; Terapias Complementares.

\section{INTRODUÇÃO}

As PIC são sistemas médicos e recursos terapêuticos complexos empregados para promoção do amplo cuidado em saúde, valorizando a autonomia, cultura e o ambiente dos indivíduos. Dessa forma, como resultado da mudança de paradigmas relacionados à assistência em saúde, da demanda popular, de recomendações da Organização Mundial de Saúde (OMS) e de diretrizes nacionais relacionadas ao tema, foi aprovada, em 2006, a Política Nacional de Práticas Integrativas e Complementares no Sistema Único

\begin{abstract}
We analyzed the scientific production, the advances, and the challenges for the implementation of Integrative and Complementary Practices (ICP) in Brazil. The study was done through an integrative review in the Virtual Health Library, selecting 68 articles. It was noted that the Southeast region continues as a scientific hub, and that most of the studies do not have a significant level of evidence. Over the years, there has been a small increase in the number of studies and greater evidence of the effectiveness and efficiency of ICP in several areas. However, problems related to poor professional training, bad management of the system, and concepts rooted in allopathic medicine remain, and hinder the expansion of ICP in Brazil.
\end{abstract}

\begin{tabular}{|c|c|c|}
\hline KEYWORDS: & Anthroposophy; & Phytotherapy; \\
\hline Homeopathy; & Traditional & Medicine \\
\hline
\end{tabular}

de Saúde SUS (PNPIC). A política tem como objetivos: a incorporação e implementação das PIC no SUS, o aumento da resolutibilidade do Sistema, a racionalização de ações de saúde e a estimulação da participação social. ${ }^{1}$

A implantação das PIC no SUS se justifica por sermos um país multicultural em que os princípios do Sistema de Saúde contemplam a integralidade da assistência e o cuidado universal. Além disso, o fácil acesso e o baixo custo da adoção dessas práticas são também fatores importantes na adesão a tratamentos e na gestão em saúde. Consequentemente, estratégias para incentivar e facilitar a

\footnotetext{
Universidade Federal de Juiz de Fora. Faculdade de Medicina. E-mail: barbarareisjf@gmail.com.

Universidade Federal de Juiz de Fora. Faculdade de Enfermagem.

Universidade Federal de Juiz de Fora. Faculdade de Enfermagem.
} 
implementação de PIC são fundamentais, porém a descoberta e a melhoria das ações se dão, principalmente, por meio da análise feita por estudos epidemiológicos que tratem do assunto. ${ }^{2}$

Em relação aos avanços que a PNPIC tem proporcionado, podem-se citar: normatização e institucionalização das PIC na rede pública; aumento significativo de ações, programas e políticas nos estados e municípios; integração dessas práticas aos sistemas oficiais de saúde; desenvolvimento de conhecimento na área, além de legislação/ normatização para oferta de serviços e produtos de qualidade; qualificação dos profissionais envolvidos com práticas complementares; melhoria da atenção à saúde da população e ampliação das opções terapêuticas aos usuários do SUS. ${ }^{3}$ Entretanto, como desafios são apresentados: a dificuldade na formação e qualificação de profissionais em número adequado para atuarem no SUS; o monitoramento e avaliação dos serviços; o fornecimento dos insumos (medicamentos homeopáticos/fitoterápicos/ agulhas para Medicina Tradicional Chinesa - Acupuntura); a estruturação dos serviços na rede pública e o investimento para desenvolvimento de processos e produtos. ${ }^{3}$

Frente a esse contexto, neste estudo, iremos analisar a conjuntura da produção científica sobre PIC no Brasil, relacionando os achados com os avanços e desafios para a implementação das práticas no país.

\section{MATERIAL E MÉTODO}

Buscando-se uma abordagem mais ampla do tema, foi realizado um estudo quanti-qualitativo por meio de pesquisa bibliográfica do tipo Revisão Integrativa na Biblioteca Virtual em Saúde, em agosto e setembro de 2015, com os termos: práticas integrativas e complementares, medicina alternativa, fitoterapia, acupuntura, medicina antroposófica ou antroposofia, termalismo ou crenoterapia e homeopatia.

Os critérios de inclusão foram: artigo disponível na íntegra, em português, publicado em 2013, 2014 e 2015. No total, foram encontradas 203 publicações. Em seguida, fez-se a leitura do título e do resumo de todos os arquivos e a montagem de uma planilha chamada Fase 0 no Excel, com as colunas: título, autor e coautores, ano, local de publicação e nome da revista. Foram excluídos: cartas ao editor, resenhas, revisões, pesquisas com animais, publicações estrangeiras, artigos duplicados, indisponíveis, não relacionados às PIC ou já selecionados previamente em pesquisa com outro termo.

Ao final dessa seleção, obtivemos 68 artigos, os quais sofreram fichamento, em uma segunda planilha do Excel, com os objetivos, método, população de estudo, resulta- dos, práticas integrativas estudadas e propostas. Assim, foi possível realizar os cálculos estatísticos para caracterizar a amostra, analisar os dados e responder às perguntas norteadoras: a pesquisa científica sobre PIC no Brasil teve incremento nos últimos 3 anos? Quais os avanços e desafios para a implementação das práticas no país?

Este trabalho é uma continuidade e atualização do estudo prévio de Greco et al., ${ }^{4}$ o qual utilizou a mesma metodologia para a realização de estudos de revisão estabelecida por Gil, ${ }^{5}$ e caracterizou-se por uma Revisão Integrativa da literatura sobre produção científica relacionada às PIC entre 2002 e 2012.

\section{RESULTADOS E DISCUSSÃO}

Dos 68 artigos selecionados, 25 são do ano de 2013, 32 de 2014 e 11 de 2015. A região do país com maior destaque foi a Sudeste, com $87 \%$ dos artigos disponibilizados, permanecendo como polo produtor científico (Figura 1) (Tabela 1).

Ao compararmos os dados deste estudo com os encontrados por Greco et al., observamos um aumento no número de publicações sobre PIC nos últimos 3 anos, uma vez que, entre os anos de 2002 a 2012, foram encontrados 49 estudos e, entre 2013 a 2015, 68 estudos.

As revistas com maior número de publicações são as que tratam especificamente de práticas integrativas ou estão relacionadas à saúde coletiva, demonstrando pouca inserção do assunto em áreas mais específicas, nas quais a medicina alopática ainda é vigente (Figura 2).

Em relação ao método, os mais citados foram: os descritivo/observacional/relato e estudo de caso/história oral/exploratório (38\%); qualitativo $(25 \%)$ e ensaio clínico/experimental (10\%). Dessa forma, observa-se que poucos são os estudos que se debruçam sobre a discussão da eficácia e segurança das PIC (Figura 3).

As práticas integrativas mais abordadas foram: Fitoterapia, Plantas Medicinais e Acupuntura, sendo importante destacar a inexistência de artigos sobre Termalismo Social e Crenoterapia (Tabela 2).

Pelos critérios do estudo, 7 mencionaram o ensino das PIC na graduação, mais especificamente sobre o aprendizado da Acupuntura, o entendimento dos estudantes acerca da Homeopatia e das PIC em geral. Também relatam as dificuldades, a importância e as perspectivas para inserção das práticas na grade curricular, as contribuições da Antroposofia para a integralidade da educação médica e as discrepâncias entre o ensino e a prática de profissionais recentemente especializados em Homeopatia. Percebe-se pouca abordagem e conhecimento das PIC no cotidiano acadêmico, muitos entraves relacionados à Me- 
dicina Baseada em Evidências e pouca valorização de uma visão holística no cuidado em saúde, justificando, em par- te, as barreiras para adoção das práticas por profissionais de saúde. ${ }^{6,7,8,9} \cdot 10,11,12$

Figura 1 - Artigos sobre PIC publicados segundo região brasileira nos anos de 2013 a 2015.



Fonte: próprio autor.

Tabela 1 - Distribuição dos artigos sobre PIC segundo a cidade de publicação nos anos de 2013 a 2015.

\begin{tabular}{lcc}
\hline Local/Cidade de publicação & $\mathbf{N}^{\mathbf{0}}$ & $\%$ \\
\hline Araraquara & 8 & 11,76 \\
Belém & 1 & 1,47 \\
Belo Horizonte & 2 & 2,94 \\
Botucatu & 11 & 16,18 \\
Brasília & 2 & 2,94 \\
Campinas & 2 & 2,94 \\
Curitiba & 3 & 4,41 \\
Fortaleza & 1 & 1,47 \\
João Pessoa & 1 & 1,47 \\
Juiz de Fora & 1 & 1,47 \\
Londrina & 1 & 1,47 \\
Marília & 1 & 1,47 \\
Rio de Janeiro & 20 & 29,41 \\
São Paulo & 14 & 20,59 \\
Total & 68 & 100,00 \\
\hline Fonte: & & \\
\hline
\end{tabular}

Fonte: próprio autor. 
Figura 2 - Distribuição dos artigos segundo a revista de sua publicação.

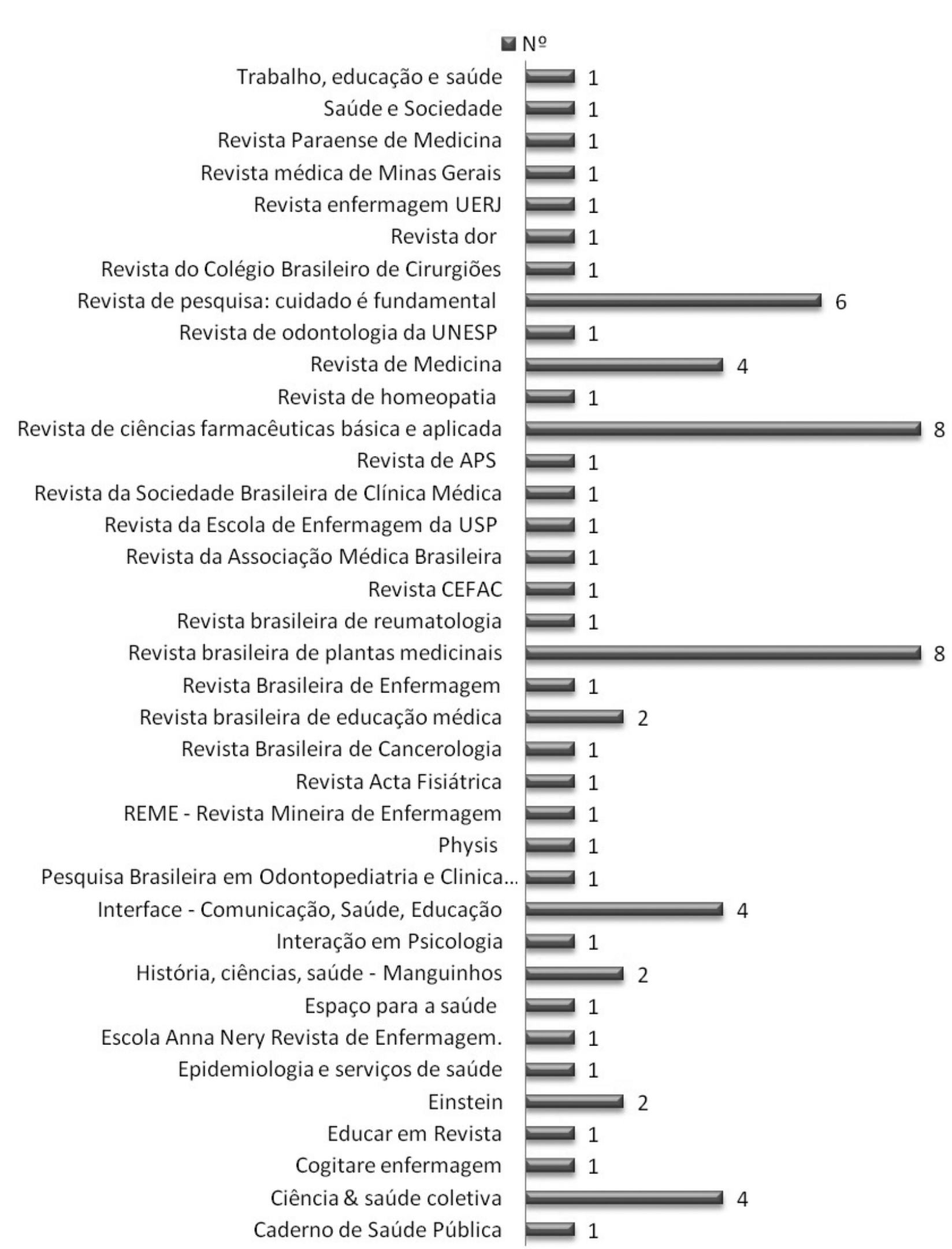

Fonte: próprio autor. 
Figura 3 - Percentual de publicações por tipo de estudo em 2013, 2014 e 2015.



Fonte: próprio autor.

Tabela 2 - Distribuição dos artigos segundo a prática integrativa abordada.

\begin{tabular}{lcc}
\hline PRÁTICAS INTEGRATIVAS & $\mathbf{N}^{\mathbf{0}}$ & $\%$ \\
\hline Acupuntura & 11 & $16,18 \%$ \\
Acupuntura e Homeopatia & 1 & $1,47 \%$ \\
Aromaterapia & 1 & $1,47 \%$ \\
Fitoterapia & 14 & $20,59 \%$ \\
Homeopatia & 8 & $11,76 \%$ \\
Homeopatia e Fitoterapia & 1 & $1,47 \%$ \\
Medicina Antroposófica & 2 & $2,94 \%$ \\
Medicina Tradicional Chinesa & 1 & $1,47 \%$ \\
Plantas Medicinais & 12 & $17,65 \%$ \\
Plantas Medicinais e Fitoterapia & 10 & $14,71 \%$ \\
Práticas Integrativas e Complementares EM GERAL & 7 & $10,29 \%$ \\
Total & 68 & $100,00 \%$ \\
\hline
\end{tabular}

Fonte: próprio autor. 
Apenas 3 artigos relatam questões relativas à inserção das PIC no Sistema Único de Saúde (SUS). Os temas englobam como organizá-las dentro do serviço, como utilizálas para promoção de saúde, como se deu a implantação e os desafios da Fitoterapia no SUS e aspectos do uso de plantas medicinais por usuários das Unidades Básicas de Saúde. Ou seja, pouco foi pesquisado e analisado sobre a conjuntura atual e futura das PIC no Brasil anos após a implementação da PNPIC, sugerindo a necessidade de uma maior autoavaliação por parte do Sistema junto à comunidade científica para correção das falhas existentes. $13,14,15$

O conhecimento dos profissionais de saúde sobre PIC foi abordado em 11 artigos. Nesse contexto, investigouse a relação existente entre a aprendizagem da Fitoterapia e atitudes na prática clínica de cirurgiões-dentistas e médicos de Unidades Básicas de Saúde, descobriram-se modelos explicativos do setor profissional em relação às plantas medicinais, dando destaque para as opiniões, vantagens e facilidades encontradas por médicos e enfermeiros no uso da Fitoterapia e plantas medicinais na atenção básica. A Técnica Delphi foi apresentada como método de intervenção pela enfermagem por meio da Acupuntura e conheceu-se a percepção de gestores em saúde e profissionais da estratégia saúde da família sobre a inserção da Fitoterapia na atenção primária. Algo muito importante foi a menção à necessidade de conhecimento sobre propriedades físico-químicas de plantas medicinais e variações sazonais para o uso adequado das PIC por profissionais de saúde e a análise da transferência dos conhecimentos adquiridos sobre Acupuntura para a atual Medicina Baseada em Evidências. Por fim, e com incontável valor humanístico, avaliou-se a relação positiva do conhecimento de PIC e dos princípios éticos dos enfermeiros com a opção pelo uso das práticas no cotidiano dos pacientes. . $^{16,17,18,19,20,21,22,23,24,25}$

Um grande número de estudos (19) trata da utilização das práticas na prevenção, promoção, assistência, tratamento e reabilitação de doenças. Por meio deles, se identificaram as práticas alternativas de cuidado utilizadas por familiares de crianças e adolescentes com infecção latente por tuberculose sob tratamento preventivo com Izoniazida, a importância de plantas medicinais como primeira linha de ação para enfermidades e o uso do óleo essencial de Yang Yang por meio de aplicação cutânea ou inalatória na ansiedade, autoestima e parâmetros fisiológicos. Além disso, estudou-se o possível efeito tóxico da associação Hypericum com a copaíba, o que é de fundamental importância para esclarecer sobre a necessidade de conhecimento técnico do tema respaldando prescrições sem interações medicamentosas. Também foram descritas as plantas medicinais utilizadas como analgésicas pela população e como se dá o uso e o preparo de medicamentos naturais pela comunidade. Ademais, estudou-se como a linha de pensamento Ying e Yang contribui na elaboração do sistema diagnóstico e terapêutico e qual é a eficácia da Acupuntura no tratamento da Fibromialgia e da dor na anemia falciforme. ${ }^{26,27,28,29,30,31,32,33,34}$

Merecem destaque pela relevância do tema associado às PIC na atualidade: a pesquisa sobre como utilizar as terapias complementares nos cuidados paliativos, o artigo relacionado à eficácia do tratamento com Paullinia cupana para controle de fogachos em sobreviventes de câncer de mama e o estudo sobre os programas e ações de Fitoterapia empregados na atenção primária à saúde brasileira desde sua implantação no sistema de saúde. . $5,36,37^{-37}$

Para a elaboração coletiva de projetos terapêuticos compartilhados, analisaram-se as possibilidades que a construção de histórias de vida em encontros organizados e as oficinas multiprofissionais de educação permanente podem oferecer para o cuidado em saúde, além das possibilidades de contribuição da Homeopatia no contexto da atenção básica. ${ }^{38,39,40}$

Revisões recentes na quimioterapia de Leishmaniose enfatizam as deficiências dos agentes terapêuticos atualmente disponíveis e mostram a necessidade urgente de novas opções de tratamento. Uma alternativa para substituir esses medicamentos, segundo um dos estudos, são os extratos naturais de Eugenia uniflora e Momordica charantia, comprovando a aplicabilidade e eficiência do uso de PIC em níveis secundários e terciários de atenção. ${ }^{41}$

Além disso, segundo Mello et al., ${ }^{42}$ as práticas religiosas constituem lugares de acolhimento, de cura e de saúde para aqueles que as buscam, não implicando necessariamente em remoção dos sintomas, mas mudança positiva dos significados atribuídos à doença e criação de rede social de apoio. Podem, assim, complementar as práticas médicas oficiais.

Outros estudos demonstraram a eficiência das PIC no tratamento de doenças crônicas e incapacitantes que demandam tratamento em longo prazo e de maior custo, como o estudo de Milcheski, ${ }^{43}$ que utilizou terapia por pressão negativa para o cuidado de feridas complexas e também o estudo que demonstrou alternativas ligadas às PIC para o tratamento da hérnia de disco lombar. ${ }^{44}$

O conhecimento da população sobre PIC é referenciado em 13 artigos, estando ligado principalmente às Plantas Medicinais para tratamento de feridas e à Acupuntura, com predomínio entre idosos e no sexo feminino. $45,46,47,48,49,50,37,51,52,53,54,55,56$

Foram encontradas 12 pesquisas experimentais com PIC, identificando-se plantas com ações antimicrobia- 
nas, laxativas, antioxidantes, analgésicas e antieméticas. ${ }^{57,58,59,60,61,62,63,64,65}$ Além disso, caracterizou-se o consagrado uso da acupuntura como ansiolítico. ${ }^{66}$ Mas um dos artigos demonstrou também falhas na qualidade e rotulagem de Fitoterápicos, indicando que, nesse aspecto, ainda temos muito que avançar. ${ }^{67}$

\section{CONCLUSÃO}

Dessa forma, observa-se que houve pequeno aumento no número de estudos nos últimos anos e maior comprovação da eficácia e eficiência das PIC em diversas áreas, algo positivo para o país e toda a ciência. Infelizmente, antigos polos de estudo predominam, sendo ainda pequeno o incentivo em áreas socioeconomicamente desfavorecidas. Os desafios atuais estão nos entraves relacionados a pouca formação profissional, má gestão do Sistema e concepções enraizadas na medicina alopática, que permanecem e dificultam a ampliação das PIC no país.

\section{REFERÊNCIAS}

1. Ministério da Saúde (Brasil). Política Nacional de Práticas Integrativas e Complementares no SUS - PNPICSUS: atitude de ampliação de acesso. $2^{a}$ ed. Brasília: Ministério da Saúde; 2015.

2. Minayo MCS, Hartz ZMA, Buss PM. Qualidade de vida e saúde: um debate necessário. Ciência e Saúde Coletiva. 2000; 5(1):7-18.

3. Ministério da Saúde (Brasil). Secretaria de Atenção à Saúde. Departamento de Atenção Básica. Coordenação Nacional de Práticas Integrativas e Complementares. Relatório de Gestão 2006/2010: Práticas Integrativas e Complementares no SUS. Brasília: Ministério da Saúde; 2011.

4. Greco RM et al. Estudos brasileiros sobre práticas integrativas e complementares. Revista Sodebras. 2016; 11(121):85-91.

5. Gil AC. Como elaborar projetos de pesquisa. $4^{a}$ ed. São Paulo: Atlas; 2007.184p.

6. Morales NM, Min LS, Teixeira JEM. Atitude de estudantes de medicina frente a terapias alternativas e complementares. Revista Brasileira de Educação Médica. 2015; 39(2):240-245.

7. Oliveira SS et al. Paullinia cupana para o controle de fogachos em pacientes com câncer de mama: um estudo piloto. Einstein. 2013; 11(4):435-438.

8. Roland MI, Gianini RJ. Redes sociotécnicas de assistência à saúde em acupuntura: estudo de caso sobre a formação básica de estudantes de medicina. História, Ciências, Saúde - Manguinhos. 2014; 21(2):477-511.

9. Sigolo RP. Ensino médico e legitimação: a presença da homeopatia na Faculdade de Medicina do Paraná nos anos 1910. Educar em Revista. 2014; (54):19-35.

10. Souza EPV, Peixoto MAP. Crenças epistemológicas e o processo de aprendizagem da homeopatia. Revista Brasileira de Educação Médica. 2015; 39(2):218-225.

11. Teixeira MZ, Lin CA. Educação médica em terapêuticas não convencionais. Revista de Medicina. 2013; 92(4):224-35.

12. Wenceslau LD, Röhr F, Tesser CD. Contribuições da medicina antroposófica à integralidade na educação médica: uma aproximação hermenêutica. Interface-Comunicação, Saúde, Educação. 2014; 18(48):127-138.

13. Araújo CRF, Silva AB, Tavares EC, Costa EP, Mariz SR. Perfil e prevalência de uso de plantas medicinais em uma unidade básica de saúde da família em Campina Grande, Paraíba, Brasil. Revista de Ciências Farmacêuticas Básica e Aplicada. 2014; 35(2):233-238.

14. Figueredo CA, Gurgel IGD, Gurgel JGD. A Política Nacional de Plantas Medicinais e Fitoterápicos: construção, perspectivas e desafios. Physis. 2014; 24(2):381-400.

15. Lima DF, Pereira DL, Franciscon FF, Reis C, Lima VS, Cavalcanti PP. Conhecimento e uso de plantas medicinais por usuários de duas unidades básicas de saúde. Revista da Rede de Enfermagem do Nordeste. 2014; 15(3):383-390.

16. Fontenele RP et al. Fitoterapia na Atenção Básica: olhares dos gestores e profissionais da Estratégia Saúde da Família de Teresina (PI). Ciência e Saúde Coletiva. 2013; 18(8):2385-2394.

17. Lin CA. Da medicina tradicional chinesa à prática de acupuntura médica baseada em evidência. Revista de Medicina. 2013; 92(3):213-215.

18. Melo SCC et al. Práticas complementares de saúde e os desafios de sua aplicabilidade no hospital: visão de 
enfermeiros. Revista Brasileira de Enfermagem. 2013; 66(6):840-846.

19. Palma JS, Badke MR, Heisler EV, HECK RM, Meincke SMK. Modelos explicativos do setor profissional em relação às plantas medicinais. Revista de Pesquisa: Cuidado é Fundamental. 2015; 7(3):2998-3008.

20. Pereira RDM, Alvim NAT. Técnica Delphi no diálogo com enfermeiros sobre a acupuntura como proposta de intervenção de enfermagem. Escola Anna Nery. 2015; 19(1):174-180.

21. Pinto LN, Flor ASSO, Barbosa WLR. Fitoterapia popular na Amazônia Paraense: uma abordagem no município de Igarapé-Mirí, estado do Pará nos anos de 2000 e 2008. Revista Ciências Farmacêuticas Básica e Aplicada. 2014; 35(2):305-311.

22. Reis LBM, Farias AL, Bollella AP, Silva HKM, Canuto MIC; Zambelli JC, et al. Conhecimentos, atitudes e práticas de Cirurgiões-Dentistas de Anápolis - GO sobre a fitoterapia em odontologia. Revista Odontologia da UNESP. 2014; 43(5):319-325.

23. Sampaio LA et al. Percepção dos enfermeiros da estratégia saúde da família sobre o uso da fitoterapia. Revista Mineira de Enfermagem. 2013; 17(1):77-85.

24. Varela DSS, Azevedo DM. Difficulties of health prefessionals facing the use of medicinal plants and fitotherapy. Revista de Pesquisa: Cuidado é Fundamental. 2013; 5(2):3588-3600.

25.Varela DSS, Azevedo DM. Opinião de médicos e enfermeiros sobre o uso da fitoterapia e plantas medicinais na Atenção Básica. Revista de APS. 2014; 17(2):204-213.

26. Agollo MC, Miszputen SJ, Diament J. Hepatotoxicidade induzida por Hypericum perforatum com possível associação a copaíba (Copaifera langsdorffii Desf): relato de caso. Einstein. 2014; 12(3):355-357.

27. Botelho NM, Brito NB, Silva NM. A utilização de plantas medicinais pela comunidade do canal da Visconde. Revista Paraense de Medicina. 2014; 28(1):63-90.

28. Coutinho BD, Dulcetti PGS. O movimento Yīn e Yáng na cosmologia da medicina chinesa. História Ciência e Saúde - Manguinhos. 2015; 22(3):797-811.
29. Gnatta JR et al. Aromatherapy with ylang ylang for anxiety and self-esteem: a pilot study. Revista da Escola de Enfermagem da USP. 2014; 48(3):492-499.

30. Marchesini Stival RS et al. Acupuntura na fibromialgia: um estudo randomizado-controlado abordando a resposta imediata da dor. Revista Brasileira de Reumatologia. 2014; 54(6):431-436.

31. Marques CVP. Acupuntura a laser no tratamento da dor em criança com anemia falciforme. Relato de caso. Revista de Dor. 2014; 15(1):70-73.

32. Pires IFB et al. Plantas medicinais como opção terapêutica em comunidade de Montes Claros, Minas Gerais, Brasil. Revista Brasileira de Plantas Medicinais. 2014; 16(2):426-433.

33. Schek G, Da Rocha GBD, Palma JS, Heck RM, Barbieri RL. Plantas medicinais utilizadas para analgesia em famílias descendentes de pomeranos no Sul do Brasil. Revista de Pesquisa: Cuidado é Fundamental. 2014; 6(3):929-937.

34. Silva AR, Sousa AI, Couto S. Práticas de cuidado empregadas no tratamento de crianças e adolescentes com infecção latente por tuberculose. Epidemiologia e Serviços de Saúde. 2014; 23(3):547-552.

35. Antonio GD, Tesser CD, Moretti-Pires RO. Contribuições das plantas medicinais para o cuidado e a promoção da saúde na atenção primária. Interface. 2013;17(46):615-633.

36. Caires JS et al. A utilização das terapias complementares nos cuidados paliativos: benefícios e finalidades. Cogitare Enfermagem. 2014; 19(3):514-520.

37. Oliveira DMS, Lucena EMP. O uso de plantas medicinais por moradores de Quixadá - Ceará. Revista Brasileira de Plantas Medicinais. 2015; 17(3):407-412.

38. Salles SAC, Ayres JRCM. A consulta homeopática: examinando seu efeito em pacientes da atenção básica. Interface. 2013; 17(45):315-326.

39. Slomp Junior H, Feuerwerker LCM, Land MGP. Educação em saúde ou projeto terapêutico compartilhado? $\mathrm{O}$ cuidado extravasa a dimensão pedagógica. Ciência \& Saúde Coletiva. 2015; 20(2):537-546.

40. Slomp Junior H, Feuerwerker LCM, Merhy EE. Histórias de vida, homeopatia e educação permanente: cons- 
truindo o cuidado compartilhado. Ciência \& Saúde Coletiva. 2015; 20(6):1795-1803.

41. Alves Santos KK, Rolón M, Vega C, Rojas De Arias A, Martins Da Costa JG, Melo Coutinho HD. Atividade leishmanicida in vitro de Eugenia uniflora e Momordica charantia. Revista de Ciências Farmacêuticas Básica e Aplicada. 2013; 34(1):47-50.

42. Mello ML, Oliveira SS. Saúde, religião e cultura: um diálogo a partir das práticas afro-brasileiras. Saúde e Sociedade. 2013; 22(4):1024-1035.

43. Milcheski DA et al. Terapia por pressão negativa na ferida traumática complexa do períneo. Revista do Colégio Brasileiro de Cirurgiões. 2013; 40 (4):312-17.

44. Carvalho LB, Oyakawa A, Martins RS, Castro PCG, Ferreira LMN, Melo JSA, et al. Associação Brasileira de Medicina Física e Re $\neg$ abilitação. Sociedade Brasileira de Neurofisiologia Clínica. Hérnia de disco lombar: tratamento. Acta Fisiatr. 2013; 20(2):75-82.

45. Baptistel AC et al. Plantas medicinais utilizadas na Comunidade Santo Antônio, Currais, Sul do Piauí: um enfoque etnobotânico. Revista Brasileira de Plantas Medicinais. 2014; 16(2):406-425.

46. Cordeiro JMP, Felix LP. Conhecimento botânico medicinal sobre espécies vegetais nativas da caatinga e plantas espontâneas no agreste da Paraíba, Brasil. Revista Brasileira de Plantas Medicinais. 2014; 16(3):685-692.

47. Dias JS, Melo AC, Silva ES. Homeopatia: percepção da população sobre significado, acesso, utilização e implantação no SUS. Revista Espaço para a Saúde. 2014; 15(2):58-67.

48. Lima KMSV, Silva KL, Tesser CD. Práticas integrativas e complementares e relação com promoção da saúde: experiência de um serviço municipal de saúde. Interface. 2014; 18(49):261-272.

49. Machado HL et al. Pesquisa e atividades de extensão em fitoterapia desenvolvidas pela Rede FitoCerrado: uso racional de plantas medicinais e fitoterápicos por idosos em Uberlândia-MG. Revista Brasileira de Plantas Medicinais. 2014; 16(3):527-533.

50. Neto FRG, Almeida GSSA, Jesus NG, Fonseca MR. Estudo etnobotânico de plantas medicinais utilizadas pela
Comunidade do Sisal no município de Catu, Bahia, Brasil. Revista Brasileira de Plantas Medicinais. 2014; 16(4):856865.

51. Silva EDC, Tesser CD. Experiência de pacientes com acupuntura no Sistema Único de Saúde em diferentes ambientes de cuidado e (des)medicalização social. Caderno de Saúde Pública. 2013; 29(11):2186-2196.

52. Silva RS, Matos LSL, Araújo EC, Paixão GPN, Costa LEL, Pereira A. Práticas populares em saúde: autocuidado com feridas de usuários de plantas medicinais. Revista Enfermagem UERJ. 2014; 22(3):389-95.

53. Silva DC, Budó MLD, Schimith MaD, Heisler EV, Simon BS, Torres GV. Utilização de plantas medicinais por pessoas com úlcera venosa em tratamento ambulatorial. Revista de Pesquisa: Cuidado é Fundamental. 2015; 7(3):2985-2997.

54. Souza Maria NCV et al. Plantas medicinais abortivas utilizadas por mulheres de UBS: etnofarmacologia e análises cromatográficas por CCD e CLAE. Revista Brasileira de Plantas Medicinais. 2013; 15(4):763-773.

55. Teixeira MZ. Protocolo de experimentação patogenética homeopática em humanos. Revista de Medicina. 2013; 92(4):242-263.

56. Vargas NRC, Ceolin TS, Andrieli DZ, Mendieta MC, Ceolin S, Heck RM. Plantas medicinais utilizadas na cicatrização de feridas por agricultores da região sul do RS. Revista de Pesquisa: Cuidado é Fundamental. 2014; 6(2):550-560.

57. Herculano R, Trecco A, Trecco A, Borges F, Borges F, Pierri E, et al. Liberação de componentes do extrato de Casearia sylvestris Swartz empregando membranas de látex natural como suporte. Revista de Ciências Farmacêuticas Básica e Aplicada. 2014; 35(1):89-95.

58. Isaia Filho C, Jung LK, Mallmann IO, Sosa FF, Rocha AR, Bueno PTB. Avaliação da eficácia terapêutica e tolerabilidade da composição de Cassia fistula e Senna alexandrina Miller em uma amostra de voluntários com constipação intestinal funcional crônica: estudo clínico randomizado com placebo. Revista da Sociedade Brasileira de Clínica Médica. 2014; 12(1):15-21.

59. Macedo JM et al. Variação sazonal nos teores de flavonoides, taninos e atividade antioxidante de Davilla Rugosa 
Poir. Revista de Ciências Farmacêuticas Básica e Aplicada. 2013; 34(4):585-590.

60. Pires CH, Paula JAM, Tresvenzol LMF, Ferri PH, De Paula JR, De Sousa Fiuza T. Composição química e atividade antimicrobiana dos óleos essenciais das folhas e flores de Callistemon viminalis (sol. ex Gaertn.) G. Don ex. Loudon (Myrtaceae). Revista de Ciências Farmacêuticas Básica e Aplicada. 2013; 34(4):597-601.

61. Sá D, Ribeiro G, Rufino L, Oliveira N, Fiorini J. Atividade antimicrobiana da Uncaria Tomentosa (Willd) D. C. Revista de Ciências Farmacêuticas Básica e Aplicada. 2014; 35(1):53-57.

62. Monteiro BVB, Melo MCN, Lima KC, Sarmento DJS. Potencial antimicrobiano dos antissépticos de uso popular Anapyon ${ }^{\circledR}$, Água Rabelo ${ }^{\circledR}$ e Malvatricin ${ }^{\circledR}$ sobre microrganismos do meio ambiente oral. Pesquisa Brasileira em Odontopediatria e Clínica Integrada. 2013; 13(4):309-314.

63. Severo AAL, Souza TP, Roli LA, Soares Sobrinho JL, Medeiros FPM, Rolim Neto PJ. Otimização das condições de extração de senosídeos por soluções hidroetanólicas das folhas de Senna alexandrina MILL empregando planejamento fatorial. Revista de Ciências Farmacêuticas Básica e Aplicada. 2013; 34(4):603-609.

64. Siegel P, Barros NF. Por que as pesquisas em oncologia integrativa são importantes? Revista Brasileira de Cancerologia. 2013; 59(2):249-253.

65. Teixeira MZ. Efeito rebote dos fármacos modernos: evento adverso grave desconhecido pelos profissionais da saúde. Revista da Associação Médica Brasileira. 2013; 59(6):629-638.

66. Alves CP, Vectore C. Uso da acupuntura em crianças com sintomatologia de estresse: um estudo exploratório. Interação em Psicologia. 2013; 17(3):247-258.

67. Soares FP, Freire NM, Souza TR. Avaliação farmacognóstica e da rotulagem das drogas vegetais boldo-do-chile (Peumus boldus Molina) e camomila (Matricaria recutita L.) comercializadas em Fortaleza, CE. Revista Brasileira de Plantas Medicinais. 2015; 17(3):468-472.

Submissão: junho de 2017

Aprovação: janeiro de 2018 